\title{
SAR image reconstruction and autofocus by compressed sensing
}

\author{
S. Uḡur ${ }^{\mathrm{a}, \mathrm{b}, *}$, O. Arıkan ${ }^{\mathrm{a}}$ \\ a Bilkent University, Electrical and Electronics Engineering Department, Ankara, Turkey \\ b Meteksan Savunma, Ankara, Turkey
}

\section{A R T I C L E I N F O}

\section{Article history:}

Available online 24 July 2012

\section{Keywords:}

SAR

Autofocus

Compressed sensing

Phase error

Total variation

Under-sampling

\begin{abstract}
A B S T R A C T
A new SAR signal processing technique based on compressed sensing is proposed for autofocused image reconstruction on subsampled raw SAR data. It is shown that, if the residual phase error after INS/GPS corrected platform motion is captured in the signal model, then the optimal autofocused image formation can be formulated as a sparse reconstruction problem. To further improve image quality, the total variation of the reconstruction is used as a penalty term. In order to demonstrate the performance of the proposed technique in wide-band SAR systems, the measurements used in the reconstruction are formed by a new under-sampling pattern that can be easily implemented in practice by using slower rate A/D converters. Under a variety of metrics for the reconstruction quality, it is demonstrated that, even at high under-sampling ratios, the proposed technique provides reconstruction quality comparable to that obtained by the classical techniques which require full-band data without any under-sampling.
\end{abstract}

(C) 2012 Elsevier Inc. All rights reserved.

\section{Introduction}

Synthetic aperture radar (SAR) systems provide high resolution images of terrain reflectivity. Typically, an airborne or spaceborne platform carries a monostatic radar system on a straight flight path, while the radar transmits and receives echoes from the area of interest. The received and digitized radar returns are coherently processed to obtain significantly higher resolution in azimuth direction that would otherwise necessitate usage of a large aperture antenna.

Because of their high resolving power, SAR systems are widely used in ground surveillance, terrain mapping and environmental monitoring applications. In military applications, SAR is primarily used in intelligence, surveillance and reconnaissance (ISR) missions. Increased demand on high-precision ISR outputs requires sub-meter or even higher resolutions from SAR images. High resolution SAR systems are becoming competitive alternatives and supplements of EO/IR cameras for ISR applications. Also, the capability to operate in adverse weather conditions makes them very appealing for surveillance applications where performance of EO/IR cameras heavily depends on atmospheric conditions.

In a typical digital SAR image reconstruction processing chain, the received analog signal is first down-converted and sampled using an A/D converter without any aliasing (Fig. 1). The sampling rate of the A/D converter depends on the bandwidth of the

\footnotetext{
* Corresponding author at: Bilkent University, Electrical and Electronics Engineering Department, Ankara, Turkey.

E-mail addresses: salih@ee.bilkent.edu.tr (S. Uḡur), oarikan@ee.bilkent.edu.tr (O. Arıkan).
}

SAR system, which determines the range resolution. The A/D converted signal is written in memory for further processing. The size of the required memory is derived from the data rate of the A/D converter and the processing power of the SAR image formation processor. The stored data is either processed in real-time by an on-board processor or transferred by a data link to a ground station where the image formation takes place.

SAR systems need accurate distance and angle information between the SAR platform and the reference point in the terrain of interest in order to establish the synthetic aperture precisely. However, due to the limited accuracy of navigational sensors, there are always some residual errors left in the estimation of the actual flight path, especially in airborne SAR applications. These uncompensated platform motion errors create uncertainties in distance and angle measurements which result in phase errors in the received SAR signal. Residual phase errors are the main cause of the degradations in high resolution SAR images, limiting achievable performance especially in the azimuth direction. These errors, depending on their nature, can cause geometric distortions, loss of resolution/contrast, decrease in SNR and can even generate spurious targets [1]. Autofocus algorithms have been proposed to overcome these phase error related degradations. There are several autofocus techniques [1-8] to estimate and correct phase errors by using dedicated algorithms on raw SAR data. These algorithms can be applied on a wide range of SAR images successfully. Once a reliable estimate for the phase error is available, the raw SAR data is corrected to obtain highly improved reconstructions.

Modern SAR systems have higher range and azimuth resolutions which necessitate large bandwidths even in excess of $500 \mathrm{MHz}$. These systems typically require higher operational 


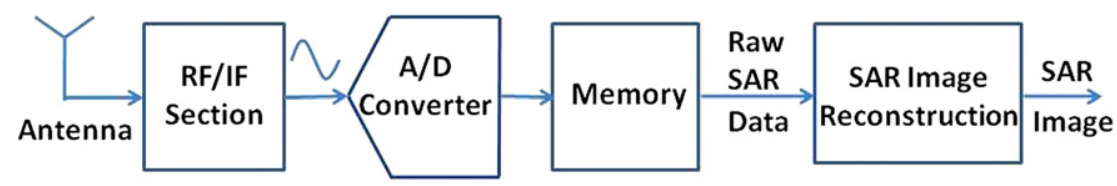

Fig. 1. Typical SAR image formation process.

frequencies because the desired bandwidths of the RF and antenna subsystems. Higher operational frequencies increase the required accuracy level of motion compensation techniques. If not compensated, these motion errors create greater phase errors. As a result, higher operational frequencies create significant challenges for autofocus techniques. Furthermore, classical image reconstruction techniques for higher frequency SAR systems require full band, fast $A / D$ converters to digitize the received signal, resulting in larger onboard storage systems. In addition, if the raw data is to be transmitted to another platform for processing, transmission of full band data requires more time or bandwidth.

Recent developments in compressed sensing (CS) techniques bring innovations to the SAR image reconstruction area. Unlike classical SAR image processing techniques, CS methods can operate on downsampled data to reconstruct SAR images where targets of interest are sparse in a certain transform domain. CS SAR image reconstruction techniques proposed in the literature model the image forming process as a sparse inverse reconstruction problem. In $[9,10]$, the inverse problem is formulated as a convex $l_{1}$ norm minimization and its solution is obtained by linear programming or approximated by greedy pursuit algorithms. The $l_{1}$ norm minimization term in the cost function of the convex minimization problem enforces sparsity. A similar approach is also used in [11] for sparse reconstruction of SAR images which uses the basis pursuit algorithm for the solution of the problem and demonstrates the super resolution property. Several computational tools are available in the literature [12,13] which can be applied to $l_{1}$ norm minimization problems. In [14], the cost function with $l_{1}$ term is solved by finding roots on the Pareto curve [12]. But they also modify the reconstruction problem by adding a total variation penalty into the cost function to reduce the speckle noise effects, which requires development of a dedicated solver. In Fourier imaging applications, the effect of $p$-norm regularization under amplitude and phase errors is studied in [15], where it is stated that for $p<2$ gradient descent optimizations reduce amplitude and phase irregularities. In [16] phase errors are accounted as SAR observation model errors and an iterative sparsity-driven method for simultaneous SAR imaging and autofocus is proposed. A coordinate descent technique is used to minimize the cost function. This method uses the fully sampled raw data to reconstruct the SAR image. Similarly, joint formulation of SAR reconstruction and autofocus has been proposed in [17], where only $50 \%$ of the Nyquist rate samples are used for the processing. There are also several other sparse SAR image reconstruction algorithms that are recently reviewed and compared in [18].

In this work, a new CS based sparse SAR image reconstruction technique is proposed. The proposed technique also performs the autofocus operation; hence it is possible to conduct image reconstruction and optimal correction of phase errors simultaneously. The proposed technique models the phase error as part of the data acquisition and then employs a CS based approach for image reconstruction and optimal phase error correction. The non-linear conjugate gradient descent algorithm is used to minimize the cost function. In addition to phase error correction, the proposed technique also incorporates total variation (TV) penalty into the cost function; hence it is called the CS-PE-TV technique. Typical CS applications rely on $l_{1}$ norm minimization approaches. However, adding TV penalty into the cost function of the SAR image reconstruction improves the quality of the output image by suppressing intensity variations caused by speckle noise, without significant effect on boundaries [14]. As a novel way of integrating both TV penalty and phase error into the cost function of the sparse SAR image reconstruction problem, the proposed technique improves the overall reconstruction quality.

To facilitate practical implementation, a new under-sampling methodology is presented in this study. It reduces the A/D conversion rate to obtain the required samples for CS SAR image reconstruction, allowing us to overcome the hardware limitations of wide band SAR applications.

The rest of the paper is organized as follows. CS is briefly reviewed in Section 2. Section 3 includes SAR data acquisition geometry and signal model. Section 4 describes the proposed undersampling methodology. The proposed technique for simultaneous reconstruction and autofocus sparse SAR images is detailed in Section 5. Section 6 provides reconstruction examples and results. Section 7 presents the conclusions. Details of the gradient computation are given in Appendix A.

\section{Compressed sensing: a short review}

Compressed sensing (CS) is a relatively new signal processing technique $[19,20]$. It can provide reliable reconstruction of a signal from its lower than Nyquist rate samples. In order to apply CS, the signal should have a sparse representation in a known basis. Since sparsity is encountered in many natural signals, CS has found diverse applications. Consider the following linear measurement model:

$y=G x$,

where $G$ is the measurement matrix, $y$ is the measured signal and $x$ is the unknown signal which can be represented sparsely in a known basis:

$x=\Psi \alpha$,

where columns of $\Psi$ are basis vectors and $\alpha$ is the vector of sparse representation coefficients. Then Eq. (1) can be written as,

$y=A \alpha$,

where $A=G \Psi$. The sparse solution for the linear model of Eq. (1) is found by solving the following $l_{0}$ norm minimization problem,

$\min \|\alpha\|_{0} \quad$ s.t. $\quad y=A \alpha$,

where $\|\alpha\|_{0}$ describes the $l_{0}$ norm, the number of non-zero entries in $\alpha$. The solution to the problem in Eq. (4) is combinatorial in nature with prohibitive computational load in practical applications. Convex relaxation of the $l_{0}$ problem to the following $l_{1}$ problem,

$\min \|\alpha\|_{1} \quad$ s.t. $\quad y=A \alpha$,

enables use of efficient programming tools for solution such as linear programming. Besides, many algorithms [21-28] to solve the convex $l_{1}$ norm minimization problems are proposed in the literature. They use various techniques including second order cone programming (SOCP), gradient descent approaches, greedy search algorithms, weighted least squares and Bregman iteration.

Uniqueness of the sparse solution of Eq. (4) is guaranteed when $\operatorname{spark}(A) / 2 \geqslant\|\alpha\|_{0}$ is satisfied [29], where spark of a matrix $A$ is 
defined as the size of the smallest linearly dependent column subset. The $\operatorname{spark}(A)$ not only controls uniqueness in Eq. (4) but also controls it in Eq. (5). No sparsity bound implies the equivalence of Eq. (4) and Eq. (5) unless $\operatorname{spark}(A) / 2 \geqslant\|\alpha\|_{0}$. A stronger condition than the spark of a matrix is the restricted isometry property (RIP) which is defined as,

$$
\begin{aligned}
& \left(1-\delta_{s}\right)\|\alpha\|_{2} \leqslant\|A \alpha\|_{2} \leqslant\left(1+\delta_{s}\right)\|\alpha\|_{2} \\
& \text { s.t. } \quad \delta_{s}>0, \quad\|\alpha\|_{0}<s,
\end{aligned}
$$

where isometry constant $\delta_{s}$ satisfies, $0 \leqslant \delta_{s}<1$ [30]. RIP provides an upper bound for the sparsity of a signal so that its energy is preserved by a given amount through the transformation by the operator. It is proven that Eq. (4) and Eq. (5) provide the same solution if $\alpha$ is sparse and $A$ holds RIP [30,31]. Unfortunately, even for moderate dimensional operators, finding RIP of a given operator is computationally impractical. However, a few classes of matrices are shown to hold RIP for almost certainly [20,30,31] which include random matrices with i.i.d. entries, Fourier ensemble matrices, and general orthogonal measurement ensembles. Note that the general orthogonal measurement ensembles can be generated by randomly selecting $n$ rows from $m$ by $m$ orthonormal matrix and re-normalizing the columns [30].

CS finds application in diverse fields thanks to its strong theoretical performance bounds. E/O and medical imaging applications benefit significantly from CS techniques due to the well known bases that are used to sparsely represent the imaged scene [32]. Applications of CS to radar and SAR are also investigated [9-11, $14,18,33-36]$. The main difficulty in applying CS to SAR imaging is finding an appropriate basis for the sparse representation of SAR images. Due to their speckle noise content, SAR images of terrain cannot be sparsely represented in known domains. Speckle noise is caused by the coherent contributions of multiple distributed reflecting objects in a resolution bin [37]; and creates difficulties in finding sparse basis for SAR images. However, for radar scenes with highly reflective man-made objects, either wavelet or standard basis vectors can be chosen for the sparse representation of the scene reflectivity distribution [38].

\section{SAR data acquisition geometry}

SAR is an active sensor system which transmits signals with large time-bandwidth product and coherently processes received signals along the flight path of the sensor platform. There are wellknown measurement models relating the received ground echo to the ground reflectance for various operational modes of the SAR system and imaging geometries [1,39]. Although the proposed CSbased sparse SAR reconstruction technique can be applied to other modes of operation, in this work we will focus on its application to spotlight mode SAR. In spotlight mode operation, the radar travels through a straight path while pointing the beam axis towards the scene center as shown in Fig. 2.

In classical SAR image reconstruction techniques, range resolution of the image depends on the bandwidth of the transmitted waveform [1,39]. Azimuth resolution is inversely proportional to the synthetic aperture length, which is related to the total observation angle in the spotlight mode SAR $[1,39]$. Assume the real part of a linear FM chirp waveform is transmitted at each azimuth position,

$s(t)= \begin{cases}e^{j\left(\omega_{0} t+a t^{2}\right)}, & |t| \leqslant \frac{T}{2} \\ 0, & \text { otherwise }\end{cases}$

where $\omega_{0}$ is the RF carrier frequency and $2 a$ is the FM rate. Each azimuth position corresponds to a different look angle, $\theta$, from the sensor platform to the center of the imaged scene. At each look

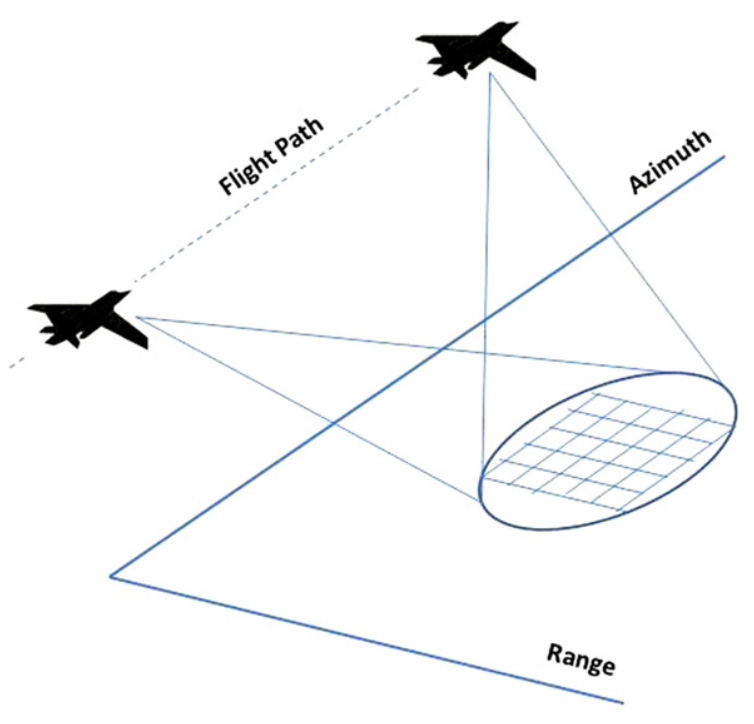

Fig. 2. Spotlight mode SAR imaging geometry.

angle, the demodulated received signal (phase history) depends on the reflectivity as [1],

$$
\begin{aligned}
r_{\theta}(t)= & \iint_{z_{1}^{2}+z_{2}^{2} \leqslant L} x\left(z_{1}, z_{2}\right) \exp \left\{-j \Omega(t)\left(z_{1} \cos (\theta)\right.\right. \\
& \left.\left.+z_{2} \sin (\theta)\right)\right\} d z_{1} d z_{2},
\end{aligned}
$$

where $L$ is the spatial extent of the ground patch and $\Omega(t)=$ $(2 / c)\left(\omega_{0}+2 a\left(t-\left(2 R_{\theta} / c\right)\right)\right)$ is the radial spatial frequency. By using the projection slice theorem, the phase history can also be written as the Fourier transform of the projection of the ground reflectivity at angle $\theta$ [40],

$r_{\theta}(t)=\int_{|u| \leqslant L} y_{\theta}(u) \exp \{-j \Omega(t) u\} d u$.

The projection of the ground reflectivity at angle $\theta$ is called the range profile. By combining Eq. (8) and Eq. (9) and discretizing the integrals in Eq. (8) using their uniform Riemann sum approximations, the discrete vector relation between the range profile at angle $\theta$ and the reflectivity of the ground patch can be written as [41],

$y_{\theta}=F_{\theta}^{-1} C_{\theta} x=G_{\theta} x$,

where $F_{\theta}^{-1}$ is the inverse discrete Fourier transform matrix and $C_{\theta}$ is the discrete approximation to the observation kernel at angle $\theta$ given in Eq. (8). Thus $G_{\theta}$ becomes the complex valued discrete SAR projection operator at angle $\theta$ relating the measured range profiles to the unknown reflectivities.

The received signal at each azimuth position of transmission as given in Eq. (10) is sampled by a high speed A/D converter. The total received signal formed through the synthetic aperture can be written in vector form by appending the range samples taken from an azimuth location to those taken from the next azimuth location. The reflectivity matrix can also be written in vector form in the same manner as stacking columns in increased order. The relation between the received signal vector and the reflectivity vector can be written as [41],

$y=G x+w$,

where $y$ is the received signal (the measurement vector), $G$ is the complex valued discrete SAR projection operator matrix, $x$ is the reflectivity vector and $w$ is the circularly symmetric white Gaussian noise vector. As indicated before, uncompensated platform 
motion errors, especially in the range direction, are the main cause of phase errors in the received signal. Phase errors can be inserted into the signal model as an exponential factor [39]:

$y_{\phi}=\Phi y=\Phi G x+w$.

Here, $\Phi$ is a diagonal matrix representing phase errors for every measurement taken from the imaged scene as given below,

$\Phi=\operatorname{diag}\{\phi\}=\left(\begin{array}{cccc}e^{j \phi_{1}} & & & \\ & e^{j \phi_{2}} & & \\ & & \ddots & \\ & & & e^{j \phi_{m}}\end{array}\right)$,

where $\phi=\left[\begin{array}{llll}e^{j \phi_{1}} & e^{j \phi_{2}} & \cdots & e^{j \phi_{m}}\end{array}\right]^{T}$ and $m$ is the total number of measurements. Hence, the phase error multiplied measurement vector $y_{\phi}$ reflects the effects of uncompensated platform motion errors. Generally phase errors do not depend on the range axis. Therefore, they are assumed to be the same for all the data corresponding to an azimuth location [39],

$$
\left(\begin{array}{c}
y_{\phi_{1}} \\
y_{\phi_{2}} \\
\vdots \\
y_{\phi_{N}}
\end{array}\right)=\left(\begin{array}{llll}
e^{j \phi_{1}} & & & \\
& e^{j \phi_{2}} & & \\
& & \ddots & \\
& & & e^{j \phi_{N}}
\end{array}\right)\left(\begin{array}{c}
G_{1} \\
G_{2} \\
\vdots \\
G_{N}
\end{array}\right) x+w
$$

where $y_{\phi_{i}}$ is the partition of the measurement vector $y_{\phi}$ which contains all the range points corresponding to the azimuth point $i$, $\phi_{i}$ is the phase error at the azimuth point $i, G_{i}$ is the partition of the matrix $G$ corresponding to the range bins of the azimuth point $i$ and $N$ is the total number of azimuth points.

\section{SAR raw data sampling for CS}

CS techniques recover an $S$-sparse signal of length $N$ with high probability if the measurement matrix satisfies RIP and the number of measurements are greater than $O(S \log (N / S))$ [42]. Reconstructing a sparse signal with fewer measurements than its corresponding Nyquist rate helps reduce the sampling rate and the amount of raw data to be processed. Reduced sampling rates allow usage of slower A/D converters in SAR systems. Furthermore the reduced amount of raw data reduces the memory requirements of the system and the necessary data link bandwidth capacity if the raw data is transferred to a ground segment for image reconstruction.

Using lower rate A/D conversion in wide bandwidth SAR has the same effect as downsampling the original data. However, regular downsampling does not generate measurement matrices with RIP [46]. RIP can be achieved by randomly discarding some of the raw data [11] or any irregular transmission along the flight path [36]. But randomly discarding some of the regularly spaced samples cannot be easily implemented in practice by using lower rate A/D converters. Instead, jittered under-sampling which controls the maximum gap in the data is proposed [43]. This undersamples data first on a coarser grid and then perturbs samples on a finer grid. Although jittered under-sampling requires accurate jitter control, it provides satisfactory results as with random under-sampling.

In this work, we propose a simple downsampling scheme that can be implemented using slower rate A/D converters, without any jitter control. In this approach, the data is first downsampled by $K$ in the range direction. The time of the initial sample at each azimuth location is pseudo-randomly chosen. This adds randomness to the data in the azimuth direction. Fig. 3 presents an example of the under-sampling pattern for $K=4$. The randomness in the range direction is obtained by randomly discarding a small ratio, $L$, of the downsampled data. This way, the prerequisites of CS are

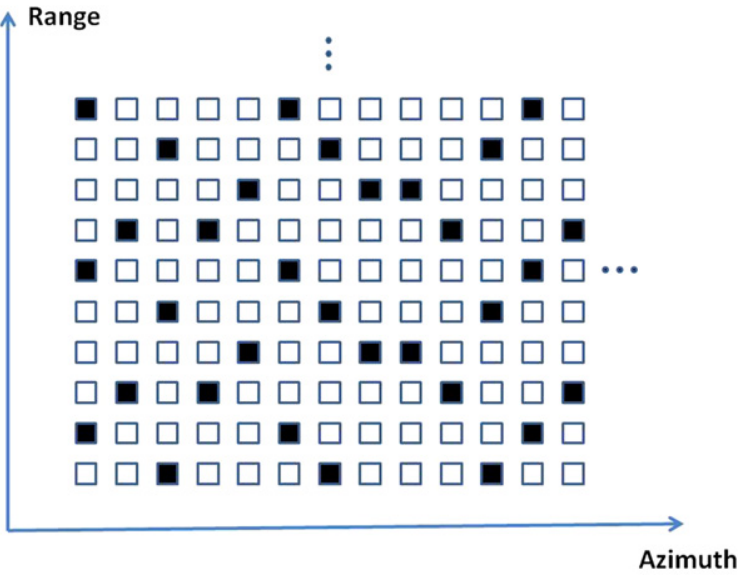

Fig. 3. Example of the proposed under-sampling pattern for $K=4$.

Table 1

A/D rate and the memory requirement for different values of parameters $K$ and $L$.

\begin{tabular}{llll}
\hline$K$ & $L$ & New A/D rate & Memory requirement \\
\hline 1 & 0 & 1 & $100 \%$ \\
2 & 0.2 & $1 / 2$ & $40 \%$ \\
3 & 0.1 & $1 / 3$ & $30 \%$ \\
4 & 0.1 & $1 / 4$ & $22.5 \%$ \\
\hline
\end{tabular}

met despite the loss of a small portion of the data. As a result, this method of under-sampling reduces the rate of the A/D converter by a factor of $K$ and reduces the memory requirement by a factor of $(1-L) / K$. Table 1 lists the reduced A/D rate and the memory requirement for different values of parameters $K$ and $L$. Note that the first row in the table is given as a reference and indicates the case of original data without any downsampling.

\section{Autofocused sparse SAR image reconstruction}

The under-sampling scheme described in Section 4 results in the following measurement relation where the number of measurements is a fraction of the number of unknowns:

$y_{u}=\Phi G_{u} x+w$,

where $y_{u}$ and $G_{u}$ represent the under-sampled measurement vector and the projection matrix respectively. Therefore, arbitrary scenes of reflectivity cannot be recovered from the available measurement data. However, if few objects with high reflectivity dominate the scene, sparse reconstruction techniques can provide reconstruction of these objects from the available data. Here $\Phi$ also corresponds to the under-sampled phase error matrix but it is not indicated as subscripted by $u$ for the sake of brevity.

Assume the transformation matrix $\Psi$ transforms the reflectivity vector $x$ to a sparse domain,

$y_{u}=\Phi G_{u} \Psi \alpha+w=\Phi A \alpha+w$.

In the presence of phase errors, sparse SAR image reconstruction can be formulated as a constrained $l_{1}$ norm minimization problem:

$\min _{\Phi, \alpha}\|\alpha\|_{1} \quad$ s.t. $\quad\left\|y_{u}-\Phi A \alpha\right\|_{2} \leqslant \sigma$

which is known as the basis pursuit denoising (BPDN) formulation. While reconstructing sparse SAR images containing man-made targets, the number of pixels a target covers can be estimated by dividing the size of target area to the area of a pixel in the reconstructed SAR image. With this estimation and the transformation in which the image is sparse, one can set an upper limit $\tau$ to the $l_{1}$-norm of the target image. Then the SAR image reconstruction 
problem can be transformed into the following Lasso formulation $[12,44]$,

$\min _{\Phi, \alpha}\left\|y_{u}-\Phi A \alpha\right\|_{2} \quad$ s.t. $\|\alpha\|_{1} \leqslant \tau$.

By integrating the phase error into the formulation, the required optimization should be carried out in terms of two sets of variables, $\alpha$ and $\Phi$. To obtain an optimal solution, Eq. (18) can be solved by first finding optimal $\Phi$ for a given $\alpha$, then transforming the problem to a minimization over $\alpha$ alone [45]. To minimize the cost in Eq. (18) for a given $\alpha$, the phase error matrix $\Phi$ that minimizes $\left\|y_{u}-\Phi A \alpha\right\|_{2}$ should be obtained. As detailed in Section 3 phase errors are assumed to be the same for all the data corresponding to an azimuth location, resulting in:

$\min \left\|y_{u}-\Phi A \alpha\right\|_{2}^{2}=\sum_{i=1}^{N} \min \left\|y_{u i}-e^{j \phi_{i}} A_{i} \alpha\right\|_{2}^{2}$,

where $y_{u i}$ is the partition of the measurement vector corresponding to the azimuth point $i$ and $A_{i}$ is the partition of the matrix $A$ corresponding to the range bins of the azimuth point $i$. Minimization of the term on the left side of the equation requires minimization of the individual terms in the summation, which can be expanded as:

$$
\begin{aligned}
\left\|y_{u i}-e^{j \phi_{i}} A_{i} \alpha\right\|_{2}^{2}= & y_{u i}^{H} y_{u i}-e^{j \phi_{i}} y_{u i}^{H} A_{i} \alpha-e^{-j \phi_{i}} \alpha^{H} A_{i}^{H} y_{u i} \\
& +\alpha^{H} A_{i}^{H} A_{i} \alpha .
\end{aligned}
$$

For each term, the minimizing $\phi_{i}$ can be found as:

$$
\begin{aligned}
\frac{\partial}{\partial \phi_{i}}\left\|y_{u i}-e^{j \phi_{i}} A_{i} \alpha\right\|_{2}^{2} & =j e^{-j \phi_{i}} \alpha^{H} A_{i}^{H} y_{u i}-j e^{j \phi_{i}} y_{u i}^{H} A_{i} \alpha \\
& =0 .
\end{aligned}
$$

The unique solution for $\phi_{i}$ can be obtained as:

$\phi_{i}=\angle\left(\alpha^{H} A_{i}^{H} y_{u i}\right)$.

With this result, Eq. (18) can be reduced to an optimization over $\alpha$ only:

$$
\min _{\alpha}\left\|y_{u}-\Phi A \alpha\right\|_{2} \quad \text { s.t. } \quad\|\alpha\|_{1} \leqslant \tau, \quad \phi_{i}=\angle\left(\alpha^{H} A_{i}^{H} y_{u i}\right) .
$$

The same approach can also be applied for the BPDN formulation given in Eq. (17). Note that, since the min-min problem is not convex, the optimization in Eq. (23) has a non-convex feasible set. Therefore, local search techniques typically converge to local minima of the problem. If the cost surface is such that the cost of the local minima is significantly higher than the global minima, a global optimization technique should be used. As illustrated in the next section, obtained results indicate that the cost of the local minima obtained by the proposed approach and the cost of the global minimum are acceptably close; therefore a slower converging global optimization technique such as particle swarm optimization is not utilized for this problem.

At this stage of the formulation, to further improve the image reconstruction quality, TV penalty is integrated into the cost function [46-50]. Integrated TV penalty smooths the target and its environment and hence reduces the noise content of the image, which also increases the effectiveness of the phase error correction. By adding TV penalty, the cost function becomes:

$$
\begin{aligned}
& \min _{x}\left\|y_{u}-\Phi G_{u} x\right\|_{2}+\beta T V(x) \text { s.t. }\left\|\Psi^{H} x\right\|_{1} \leqslant \tau, \\
& \phi_{i}=\angle\left(x^{H} G_{u i}^{H} y_{u i}\right) .
\end{aligned}
$$

Note that the relations $\alpha=\Psi^{H} x$ and $A \alpha=G_{u} x$ are used. Here, TV stands for total variation and defined as,

$T V(x)=\sum_{i, j} \sqrt{\left|\nabla_{i} x\right|_{i, j}^{2}+\left|\nabla_{j} x\right|_{i, j}^{2}}$, where subscripts $i, j$ denote pixel locations in a two dimensional image. $\nabla_{i}$ and $\nabla_{j}$ denote discrete gradients of the image in two directions and are given by,

$\left(\nabla_{i} x\right)_{i, j}=x(i, j)-x(i-1, j)$,

$\left(\nabla_{j} x\right)_{i, j}=x(i, j)-x(i, j-1)$.

The constrained optimization problem of Eq. (24) can be written as an unconstrained problem in the Lagrangian form:

$$
\begin{aligned}
& \arg \min _{x} g(x)=\left\|\Psi^{H} x\right\|_{1}+\beta T V(x)+\gamma\left\|y_{u}-\Phi G_{u} x\right\|_{2}^{2} \\
& \text { s.t. } \quad \phi_{i}=\angle\left(x^{H} G_{u i}^{H} y_{u i}\right), \quad 1 \leqslant i \leqslant N .
\end{aligned}
$$

By using the non-linear conjugate gradient descent algorithm with backtracking line search [46,51], a numerical solution to Eq. (28) can be obtained. There are more efficient algorithms published in the literature to solve $l_{1}$ minimization problems. The ones using Bregman methods are especially promising [52-54]. However, in all these techniques, the transformation in the data fidelity part of the cost function is a known matrix. But in our case, that matrix contains the unknown $x$, due to the multiplication by the phase error $\Phi$, which leads us to use the conjugate gradient method. As part of the optimization iterations, the required gradient of the cost function can be computed as detailed in Appendix A. Profiling Matlab [55] implementation of this numerical approach revealed that most of the CPU time is consumed by multiplications of large scale matrices. Therefore, by exploiting the structure of the involved matrices, significant computational improvements can also be achieved [56,57].

\section{Results obtained on synthetic and real SAR data}

In this section, results on both synthetic and real SAR data, obtained from MSTAR database [58], are presented. Raw SAR data is under-sampled as explained in Section 4. Two different setups are used for the sparse reconstructions of the synthetic and the MSTAR data. For the synthetic data, an A/D converter with $1 / 3$ of the original rate is used and $L$ is chosen as 0.1 , which results in $70 \%$ memory reduction. For the MSTAR data, half rate A/D converter is used and $L$ is chosen as 0.2 , which results in $60 \%$ memory reduction. Hence, synthetic data results are obtained by using only $30 \%$ of the original raw data while the MSTAR results are obtained by using only $40 \%$ of the original raw data.

The parameters $\beta$ and $\gamma$ in Eq. (28) can be used to adjust the relative weights of the different components in the cost function. For example, increasing the weight of the TV part smooths the noisy areas containing sudden changes in the image. Because this work is not aimed to put forward specific properties in the images, equal weights $(\beta=\gamma=1)$ are chosen for balanced contribution of different parts in the cost function unless otherwise specified.

\subsection{Synthetic data reconstructions}

The synthetic image used in the trials is shown in Fig. 4(a). Reconstructed images of the artificial scene by polar format algorithm (PFA) [1] for the same phase error, but at two different noise levels are shown in Fig. 4(b) and Fig. 4(c) respectively. Both of these reconstructions are obtained using 3 times more data than the proposed sparse reconstruction technique.

In synthetic data experiments, the transformation $\Psi$, which maps $x$ to the sparsity domain, is chosen as the identity matrix. This works quite well when the speckle noise is negligible. However, the reconstruction performance degrades when the speckle noise increases since noise starts having large projection on the impulsive basis components. 
(a)

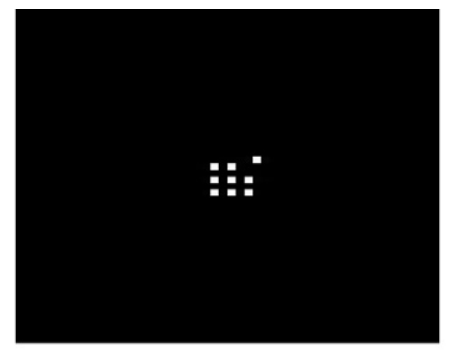

(b)

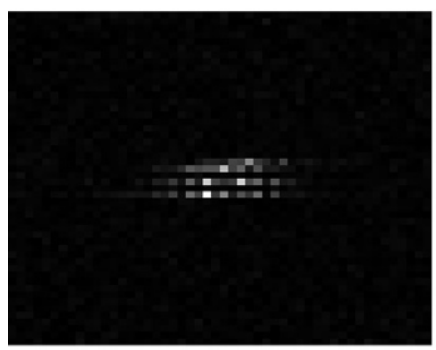

(d)
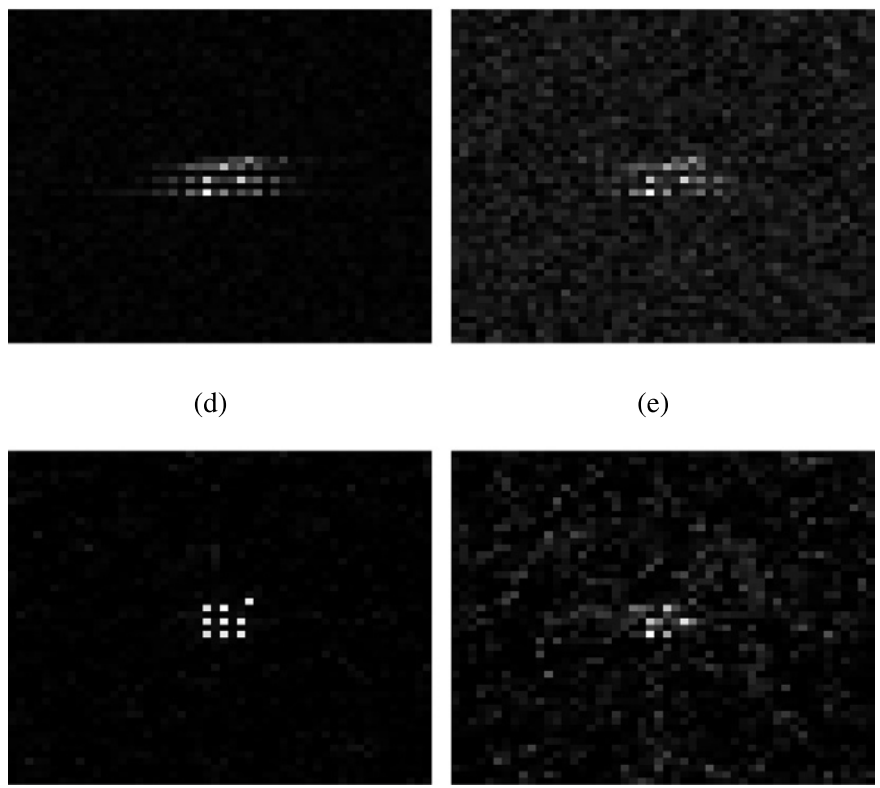

(e)

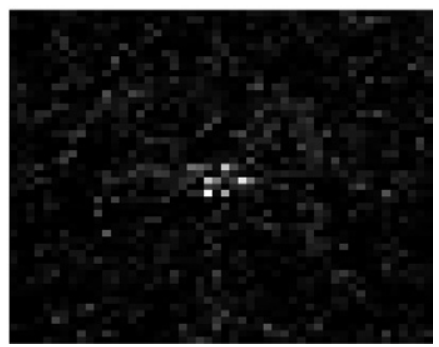

Fig. 4. The synthetic images by PFA and the reconstructions by CS-PE-TV are illustrated. (a) Original reflectivity image. (b) Image with phase error and speckle noise $(\mathrm{SNR}=31 \mathrm{~dB})$ reconstructed by PFA. (c) Image with phase error and speckle noise $(\mathrm{SNR}=19 \mathrm{~dB})$ reconstructed by PFA. (d) Image with phase error and speckle noise $(\mathrm{SNR}=31 \mathrm{~dB})$ reconstructed by CS-PE-TV. (e) Image with phase error and speckle noise $(\mathrm{SNR}=19 \mathrm{~dB})$ reconstructed by CS-PE-TV.

The obtained sparse reconstructions are shown in Fig. 4(d) and Fig. 4(e). In the case with higher SNR (Fig. 4(d)), CS-PE-TV works quite well, almost completely removing the phase error. In the case with lower SNR (Fig. 4(e)), CS-PE-TV concentrates much of the energy of the target and corrects phase error effects very well. However, it does not wholly reconstruct the original target and has some artifacts left in the scene. Compared to the case with higher SNR, the main reason for the performance degradation in the case with lower SNR is the speckle noise, which starts having larger projections on the sparse basis components.

\subsection{MSTAR reconstructions}

MSTAR database [58] contains publicly available SAR data of several target types. The ones used in the reconstruction trials are illustrated in row (a) of Fig. 5. Each contains a target in an environment with high speckle noise. These images are reconstructed using PFA. Row (b) of Fig. 5 gives the same images with artificially inserted phase error. They are reconstructed again by PFA. The applied phase error characteristics have been given in Fig. 7.

Row (c) of Fig. 5 shows the images reconstructed by PFA and autofocused by the phase gradient algorithm (PGA) [7,8]. PGA first center shifts the brightest pixels in each range bin, then applies windowing to suppress the effects of other possible targets in the same range bin. Finally, it estimates the phase error in the azimuth dimension and applies phase correction. Generally, one to three iterations of the PGA are sufficient to estimate and correct the phase errors successfully.

The results obtained by the proposed sparse SAR image reconstruction technique on the MSTAR data are illustrated in Fig. 6. For CS reconstructions, only a fraction of the whole data, obtained by the method explained in Section 4, is used. Since wavelet frames are appropriate for representing SAR images containing man-made targets, Daubechies- 4 wavelets are used as the sparsity transform in the trials with MSTAR data.

For comparison purposes, row (a) of Fig. 6 shows the results of sparse reconstruction with no phase error correction, which will be referred to as CS reconstructions. Row (b) displays the results for the case of applying autofocused sparse reconstruction without a TV penalty, which will be referred to as CS-PE reconstructions. This reconstruction is obtained by solving Eq. (28) with $\beta=0$. The results of the proposed CS-PE-TV reconstructions are presented in row (c) of Fig. 6.

As expected, CS reconstructions suffer from significant degradation due to uncompensated phase error. The images reconstructed by CS-PE show improved phase error correction compared to CS results. But the noisy nature of the images prevents further improvements. Adding TV penalty into the cost function improves the overall image reconstruction quality and success of phase error correction. This deduction is clearly demonstrated by comparing the results of CS-PE-TV to the results of CS-PE. The CS-PE-TV reconstructs the images and autofocuses them simultaneously with success.

The phase error applied to the images and its estimate obtained by the application of CS-PE-TV are shown in Fig. 7. The estimated phase error closely follows the general form of the applied phase error. The root mean square error of the estimation is only $1.9 \%$ of the radar's wavelength.

In order to quantify and compare the image reconstruction performance of the proposed CS-PE-TV technique especially on the MSTAR data, the following metrics are used:

1. Mean square error (MSE) which is defined as [59]:

$$
M S E=\frac{1}{N^{2}}\||\mathbf{x}|-|\hat{\mathbf{x}}|\|_{2}^{2},
$$

where $\mathbf{x}$ is the original image, $\hat{\mathbf{x}}$ is the reconstructed image, and $N^{2}$ is the total number of pixels in the image.

2. Target to background ratio (TBR) $[59,60]$ : This is the ratio of the absolute maximum of the target region to the absolute average of the background region. It gives an indication of how target pixels are discernible with respect to background pixels:

$T B R=20 \log _{10}\left(\frac{\max _{i \in T}\left(\left|(\mathbf{x})_{i}\right|\right)}{\frac{1}{N_{B}} \sum_{j \in B}\left|(\mathbf{x})_{j}\right|}\right)$,

where $\mathbf{x}$ is the image and $N_{B}$ is the number of pixels in the background region of the image. $T$ and $B$ represent target and background regions respectively.

3. Entropy of the image [59]: This is a metric related to sharpness of the image:

$$
H(\mathbf{x})=-\sum_{i} p_{i} \log _{2} p_{i},
$$

where the discrete variable $p$ contains the histogram counts of the image $\mathbf{x}$. Entropy is small for sharper images so it is preferable for an algorithm to result in low entropies for image formation.

These metrics give indications about the performance of the reconstructions especially on the target classification applications. 
(1)

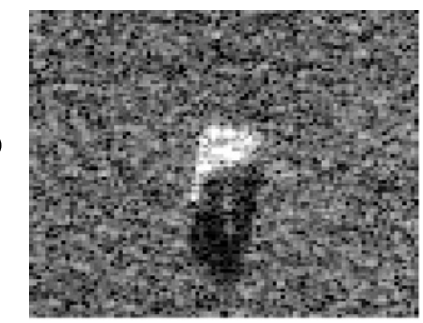

(b)

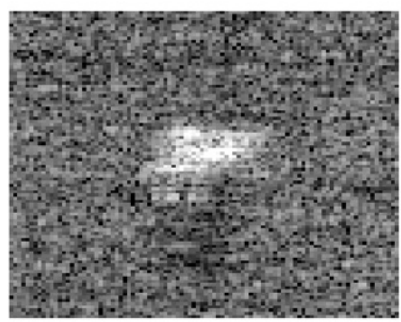

(c)

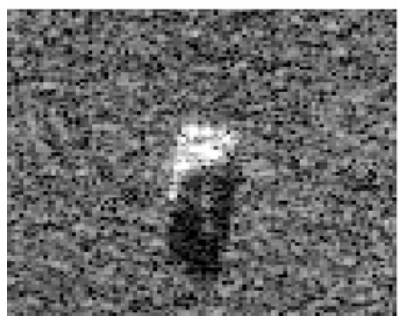

(2)
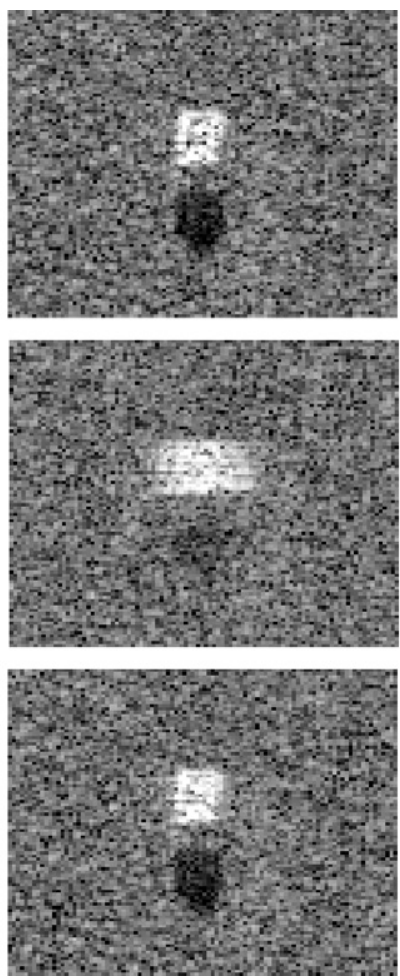

(3)
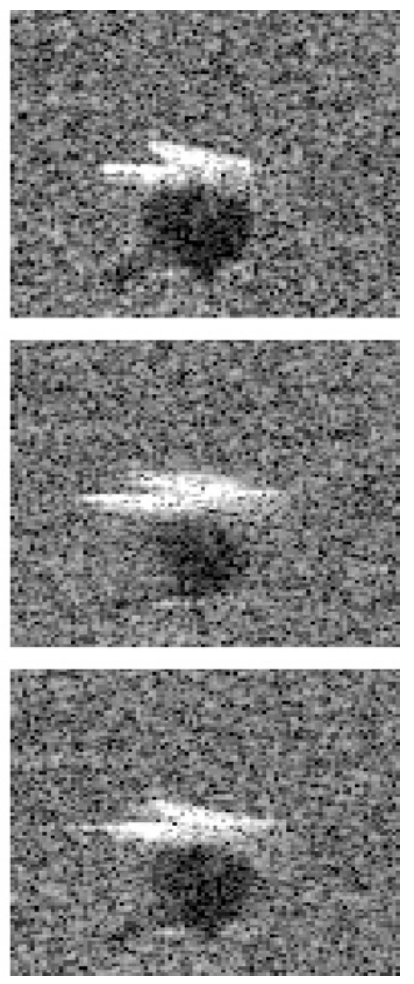

Fig. 5. Three target images of MSTAR database that are used in the trials. All the images are reconstructed by PFA. Row (a) gives the original images. Row (b) presents the images with inserted phase error. Row (c) shows the reconstructions of images autofocused by PGA.

(1)

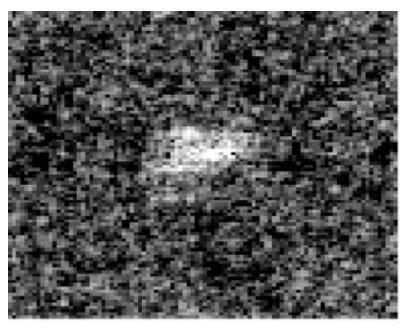

(b)

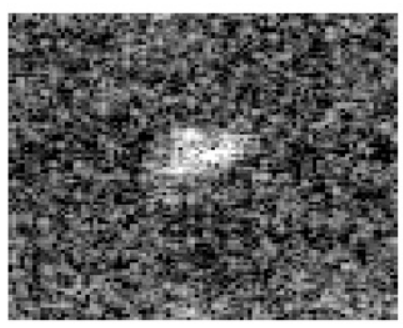

(c)

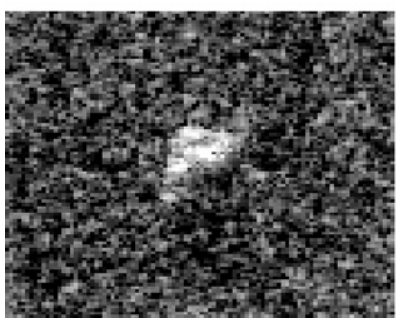

(2)
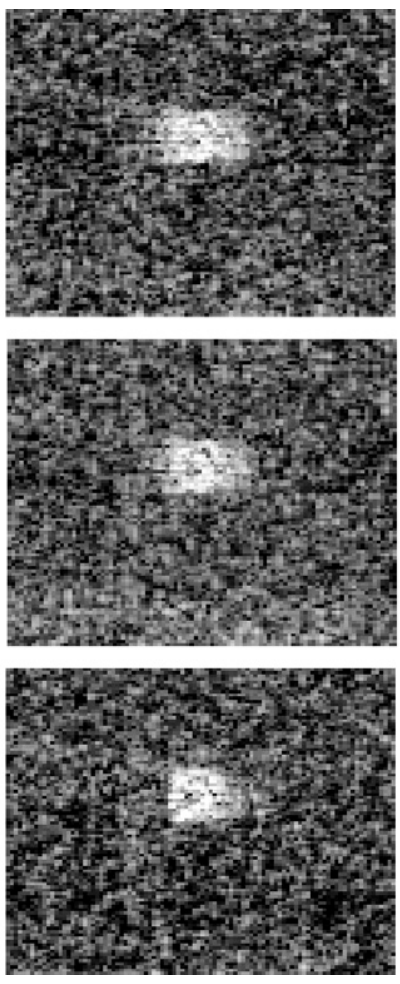

(3)
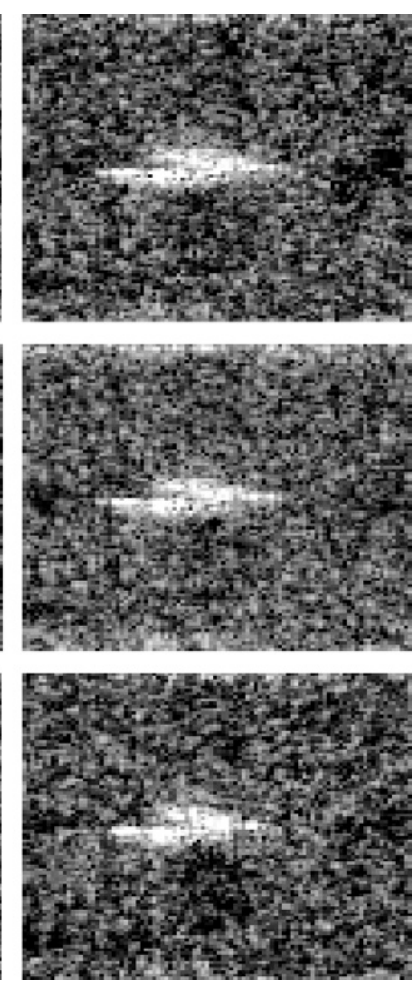

Fig. 6. Three target images of MSTAR database that are used in the trials. Row (a) gives the results of CS reconstructions. Row (b) presents the results of CS-PE reconstructions. Row (c) gives the images reconstructed by CS-PE-TV. 


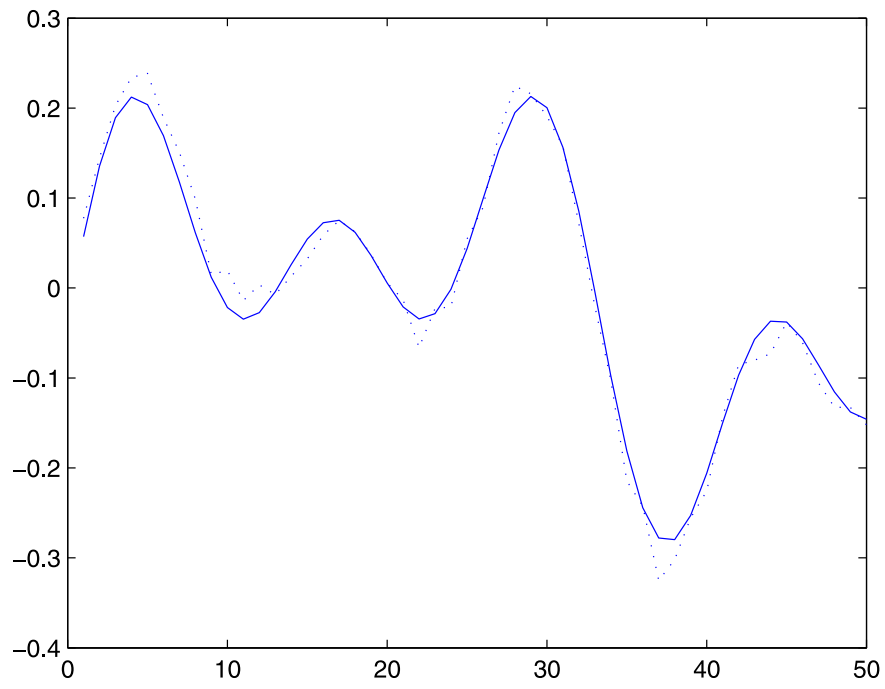

Fig. 7. The phase error applied to the image (solid line) and the phase error estimate (dotted line). $Y$-axis units represent the fractions of the SAR wavelength.

The quantitative performance metrics for the images illustrated in Fig. 6 are given in Table 2. The table lists the results for three targets which are reconstructed by using five different techniques. For each target the first row lists the metrics of the images reconstructed by PFA. The second row lists the results for the images reconstructed by PFA and autofocused by PGA. The results of the CS, CS-PE and CS-PE-TV techniques are listed in the third, fourth and fifth rows respectively. Note that these results can be improved by post processing the images. However, we are interested in comparing the performances of the applied methods alone, so no post processing is performed on the images.

PFA-PGA, which makes use of data obtained at Nyquist sampling rate, gives the best results in terms of the MSE metric. For the TBR and entropy metrics the CS-PE-TV technique provides better results than the PFA-PGA technique. The main disadvantage of the CS-PE-TV method compared to the PFA-PGA method is the computational complexity which currently limits the application of the CS-PE-TV technique to offline reconstructions. However, with the rapid advances in signal processor hardware, the application of CS to SAR image reconstruction can become feasible in the near future.

PFA performs poorly by all metrics. Even the CS technique performs better than the PFA. This result confirms the statement that $p$-norm regularizations reduce the amplitude and phase irregularities for $p<2$ [15]. The CS-PE is an improvement over CS for the MSE metric. Phase error model integration to the problem tries to compensate the effects of the phase error and guarantees more localized target images. But TBR and the entropy of the image degrade with CS-PE as compared to CS. Because the lack of TV penalty in the CS-PE technique increases the entropy. TV penalty, in the CS technique smooths the constant areas and thereby reduces the average of the background region in the TBR formulation.

With phase error model integrated and the TV penalty added, CS-PE-TV outperforms all the other techniques (except the PFAPGA technique in MSE parameter as indicated above). Almost all the metrics listed in the table indicate an improvement for the images reconstructed by the CS-PE-TV technique. Therefore, adding phase error in the signal model embeds autofocus property into the image reconstruction. Furthermore, adding in TV penalty to the cost function improves the overall reconstruction performance for SAR images with high speckle noise. In conclusion, these results demonstrate that the proposed CS-PE-TV technique provides robust, simultaneous SAR image reconstruction and autofocus.
Table 2

Performance metrics for the imagery reconstructed by different methods.

\begin{tabular}{llrll}
\hline & & \multicolumn{1}{l}{ MSE } & TBR & Entropy \\
\hline Target (1) & PFA & $6.2 \times 10^{-3}$ & 27.02 & 2.88 \\
& PFA-PGA & $\mathbf{2 . 5} \times \mathbf{1 0}^{-\mathbf{4}}$ & 29.55 & 2.50 \\
& CS & $5.7 \times 10^{-3}$ & 30.68 & 2.50 \\
& CS-PE & $5.2 \times 10^{-3}$ & 28.91 & 2.76 \\
& CS-PE-TV & $3.6 \times 10^{-3}$ & $\mathbf{3 1 . 5 9}$ & $\mathbf{2 . 4 2}$ \\
Target (2) & PFA & $7.7 \times 10^{-3}$ & 23.99 & 3.34 \\
& PFA-PGA & $\mathbf{1 . 9} \times \mathbf{1 0}^{-\mathbf{3}}$ & 27.08 & 2.95 \\
& CS & $6.8 \times 10^{-3}$ & 27.56 & 3.01 \\
& CS-PE & $6.0 \times 10^{-3}$ & 25.65 & 3.29 \\
Target (3) & CS-PE-TV & $4.5 \times 10^{-3}$ & $\mathbf{2 8 . 4 1}$ & $\mathbf{2 . 9 4}$ \\
& PFA & $10.9 \times 10^{-3}$ & 24.31 & 2.96 \\
& PFA-PGA & $\mathbf{9 . 3} \times \mathbf{1 0}^{-\mathbf{4}}$ & 26.23 & 2.80 \\
& CS & $10.4 \times 10^{-3}$ & 27.48 & 2.73 \\
& CS-PE & $9.8 \times 10^{-3}$ & 24.77 & 3.13 \\
& CS-PE-TV & $7.2 \times 10^{-3}$ & $\mathbf{3 0 . 2 3}$ & $\mathbf{2 . 4 2}$ \\
\hline
\end{tabular}

\section{Conclusions}

A compressive sensing framework is proposed for simultaneous motion compensation and image reconstruction for SAR data obtained at a fraction of Nyquist rate of sampling. Under-sampling is performed by using reduced rate $A / D$ converters which also decrease the required memory size and the bandwidth of the data link. To improve performance, a total variation penalty on the reconstructed image is also incorporated into the proposed optimization technique. Detailed investigation of the reconstructions of both synthetic and real data sets demonstrates that, under well recognized performance metrics, the proposed CS-PE-TV technique improves performance over the common PFA-PGA technique and other alternative CS based techniques.

Research on the more efficient optimization of the proposed CS-PE-TV cost function is expected to reduce the computational requirements of the proposed technique and increase its applicability.

\section{Appendix A. Gradient of the cost function}

The first term of the cost function in Eq. (28) includes absolute values. The absolute value is not a smooth function so it is approximated by,

$|x|=\sqrt{x x^{*}+\epsilon}$,

where $\epsilon$ is a small positive smoothing parameter. By using this approximation, the gradient of the first term in the cost function given in Eq. (28) becomes,

$\nabla\left\|\Psi^{H} x\right\|_{1}=\Psi W_{1}^{-1} \Psi^{H} x$,

where $W_{1}$ is a diagonal matrix with corresponding elements,

$w_{i}=\sqrt{\left|\left(\Psi^{H} x\right)_{i}\right|^{2}+\epsilon_{1}}$.

Since the total variation is also a non-smooth function, the following approximation is used,

$T V(x)=\sum_{i, j} \sqrt{\left|\nabla_{i} x\right|_{i, j}^{2}+\left|\nabla_{j} x\right|_{i, j}^{2}+\mu}$,

where $\mu$ is a small positive smoothing parameter. With this approximation, the gradient of the total variation part of the cost function is given by [49],

$\nabla T V(x)=I_{i, j}-I_{i+1, j}+J_{i, j}-J_{i, j+1}$,

where

$I_{i, j}=\frac{\left(\nabla_{i} x\right)_{i, j}}{\sqrt{\left(\nabla_{i} x\right)_{i, j}^{2}+\left(\nabla_{j} x\right)_{i, j}^{2}+\mu}}$, 


$$
J_{i, j}=\frac{\left(\nabla_{j} x\right)_{i, j}}{\sqrt{\left(\nabla_{i} x\right)_{i, j}^{2}+\left(\nabla_{j} x\right)_{i, j}^{2}+\mu}} .
$$

The third term in the cost function is re-written below,

$$
\begin{aligned}
\left\|y_{u}-\Phi G_{u} x\right\|_{2}^{2}= & y_{u}^{H} y_{u}+x^{H} G_{u}^{H} G_{u} x-y_{u}^{H} \Phi G_{u} x \\
& -x^{H} G_{u}^{H} \Phi^{H} y_{u} .
\end{aligned}
$$

The last two terms of the above equation can be expanded as,

$$
\begin{aligned}
\left\|y_{u}-\Phi G_{u} x\right\|_{2}^{2}= & y_{u}^{H} y_{u}+x^{H} G_{u}^{H} G_{u} x \\
& -\sum_{i=1}^{N} e^{j \phi_{i}} y_{u i}^{H} G_{u i} x-\sum_{i=1}^{N} e^{-j \phi_{i}} x^{H} G_{u i}^{H} y_{u i} .
\end{aligned}
$$

Putting the relation $\phi_{i}=\angle\left(x^{H} G_{u i}^{H} y_{u i}\right)$ which is obtained by Eq. (22) into the above equation,

$$
\left\|y_{u}-\Phi G_{u} x\right\|_{2}^{2}=y_{u}^{H} y_{u}+x^{H} G_{u}^{H} G_{u} x-2 \sum_{i=1}^{N}\left|y_{u i}^{H} G_{u i} x\right| .
$$

By using the approximation in Eq. (A.1), the gradient of the third term of the cost function is given by,

$$
\begin{aligned}
\nabla & \left\|y_{u}-\Phi G_{u i} x\right\|_{2}^{2} \\
& =2 G_{u}^{H} G_{u} x-2 \sum_{i=1}^{N} \frac{y_{u i}^{H} G_{u i} x}{\sqrt{x^{H} G_{u i}^{H} y_{u i} y_{u i}^{H} G_{u i} x+\epsilon_{2}}} G_{u i}^{H} y_{u i} .
\end{aligned}
$$

By combining gradients of all these three terms, the following final expression for the gradient of the cost function is obtained:

$$
\begin{aligned}
& \nabla g(x)=\Psi W_{1}^{-1} \Psi^{H} x+\beta\left(I_{i, j}-I_{i+1, j}+J_{i, j}-J_{i, j+1}\right) \\
& \quad+\gamma\left(2 G_{u}^{H} G_{u} x-2 \sum_{i=1}^{N} \frac{y_{u i}^{H} G_{u i} x}{\sqrt{x^{H} G_{u i}^{H} y_{u i} y_{u i}^{H} G_{u i} x+\epsilon_{2}}} G_{u i}^{H} y_{u i}\right) .
\end{aligned}
$$

\section{References}

[1] W.G. Carrara, R.S. Goodman, R.M. Majewski, Spotlight Synthetic Aperture Radar, Signal Processing Algorithms, Artech House Inc., 1995.

[2] C.E. Mancill, J.M. Swiger, A mapdrift autofocus technique for correcting higher order SAR phase errors, in: 27th Annual Tri-Service Radar Symposium Record, Monterey, CA, 1981, pp. 391-400.

[3] L. Xi, L. Guosui, J. Ni, Autofocusing of ISAR images based on entropy minimization, IEEE Trans. Aerospace Electron. Syst. 35 (4) (1999) 1240-1252.

[4] C.V. Jakowatz Jr., D.E. Wahl, Eigenvector method for maximum-likelihood estimation of phase errors in synthetic-aperture-radar imagery, J. Opt. Soc. Am. A 10 (12) (1993) 2539-2546.

[5] R.L. Morrison, M.N. Do, D.C. Munson, SAR image autofocus by sharpness optimization: a theoretical study, IEEE Trans. Image Process. 16 (9) (2007) 23092321.

[6] R.L. Morrison, M.N. Do, D.C. Munson, MCA: a multichannel approach to SAR autofocus, IEEE Trans. Image Process. 18 (4) (2009) 840-853.

[7] P.H. Eichel, D.C. Ghiglia, C.V. Jakowatz, Speckle processing method for synthetic aperture radar phase correction, Opt. Lett. 14 (1989) 1-3.

[8] D.E. Wahl, P.H. Eichel, D.C. Ghiglia, C.V. Jakowatz, Phase gradient autofocus a robust tool for high resolution SAR phase correction, IEEE Trans. Aerospace Electron. Syst. 30 (1994) 827-835.

[9] R. Baraniuk, P. Steeghs, Compressive radar imaging, in: IEEE Radar Conference, April 2007, pp. 128-133.

[10] S. Bhattacharya, T. Blumensath, B. Mulgrew, M. Davies, Fast encoding of synthetic aperture radar raw data using compressed sensing, in: IEEE/SP 14th Workshop on Statistical Signal Processing, August 2007, pp. 448-452.

[11] S.J. Wei, X.L. Zhang, J. Shi, G. Xiang, Sparse reconstruction for SAR imaging based on compressed sensing, Progr. Electromagn. Res. 109 (2010) 63-81.

[12] E. van den Berg, M.P. Friedlander, Probing the Pareto frontier for basis pursuit solutions, SIAM J. Sci. Comput. 31 (2) (2009) 890-912.

[13] E. Candès, J. Romberg, http://www-stat.stanford.edu/ candes/11 magic.
[14] V.M. Patel, G.R. Glenn, D.M. Healy, R. Chellappa, Compressed synthetic aperture radar, IEEE J. Sel. Top. Signal Process. 4 (2) (2010) 244-254.

[15] B.D. Rigling, Use of nonquadratic regularization in Fourier imaging, IEEE Trans. Aerospace Electron. Syst. 45 (1) (2009) 250-264.

[16] G.Ö. Önhon, M. C̣etin, Joint sparsity-driven inversion and model error correction for radar imaging, in: IEEE International Conference on Acoustics Speech and Signal Processing, March 2010, pp. 1206-1209.

[17] S.I. Kelly, G. Rilling, M. Davies, B. Mulgrew, Iterative image formation using fast (re/back)-projection for spotlight-mode SAR, in: 2011 IEEE Radar Conference, May 2011, pp. 835-840.

[18] L.C. Potter, E. Ertin, J.T. Parker, M. Cetin, Sparsity and compressed sensing in radar imaging, Proc. IEEE 98 (6) (2010) 1006-1020.

[19] D.L. Donoho, Compressed sensing, IEEE Trans. Inform. Theory 52 (4) (2006) 1289-1306.

[20] E. Candès, T. Tao, Near-optimal signal recovery from random projections: universal encoding strategies?, IEEE Trans. Inform. Theory 52 (12) (2006) 54065425

[21] S.S. Chen, D.L. Donoho, M.A. Saunders, Atomic decomposition by basis pursuit, SIAM J. Sci. Comput. 20 (1) (1998) 33-61.

[22] S. Mallat, Z. Zhang, Matching pursuits with time-frequency dictionaries, IEEE Trans. Signal Process. 41 (12) (1993) 3397-3415

[23] J.A. Tropp, Greed is good: algorithmic results for sparse approximation, IEEE Trans. Inform. Theory 50 (10) (2004) 2231-2242.

[24] J.A. Tropp, Just relax: convex programming methods for identifying sparse signals in noise, IEEE Trans. Inform. Theory 52 (3) (2006) 1030-1051.

[25] R. Baraniuk, V. Cevher, M. Duarte, C. Hedge, Model-based compressive sensing, IEEE Trans. Inform. Theory 56 (4) (2010) 1982-2001.

[26] J.A. Tropp, A.C. Gilbert, Signal recovery form random measurements via orthogonal matching pursuit, IEEE Trans. Inform. Theory 53 (12) (2007) 4655-4666.

[27] I.F. Gorodnitsky, B.D. Rao, Sparse signal reconstruction from limited data using FOCUSS: a re-weighted minimum norm algorithm, IEEE Trans. Signal Process. 45 (3) (1997) 600-616.

[28] W. Yin, S. Osher, D. Goldfarb, J. Darbon, Bregman iterative algorithms for $l_{1}$ minimization with applications to compressed sensing, SIAM J. Imaging Sci. 1 (2008) 143-168.

[29] D.L. Donoho, M. Elad, Optimally sparse representation in general (nonorthogonal) dictionaries via L1 minimization, Proc. Natl. Acad. Sci. USA 100 (2003) 2197-2202.

[30] E. Candès, J. Romberg, T. Tao, Stable signal recovery from incomplete and inaccurate measurements, Comm. Pure Appl. Math. 59 (8) (2006) 1207-1223.

[31] E. Candès, J. Romberg, T. Tao, Robust uncertainty principles: exact signal reconstruction from highly incomplete frequency information, IEEE Trans. Inform. Theory 52 (2) (2006) 489-509.

[32] E. Candès, M.B. Wakin, An introduction to compressive sampling, IEEE Signal Process. Mag. 25 (2) (2008) 21-30.

[33] M. Herman, T. Strohmer, Compressed sensing radar, in: IEEE Radar Conference, May 2008, pp. 1-6.

[34] M.A. Herman, T. Strohmer, High resolution radar via compressed sensing, IEEE Trans. Signal Process. 57 (6) (2009) 2275-2284.

[35] G.E. Smith, T. Diethe, Z. Hussain, J.S. Taylor, D.R. Hardoon, Compressed sampling for pulse Doppler radar, in: IEEE Radar Conference, May 2010, pp. 887-892.

[36] Q. Huang, L. Qu, B. Wu, G. Fang, UWB through-wall imaging based on compressive sensing, IEEE Trans. Geosci. Remote Sens. 48 (3) (2010) 1408-1415.

[37] C. Oliver, S. Quegan, Understanding Synthetic Aperture Radar Images, SciTech Publishing, 2004

[38] G. Rilling, M. Davies, B. Mulgrew, Compressed sensing based compression of SAR raw data, in: SPARS'09 - Signal Processing with Adaptive Sparse Structured Representations, March 2010.

[39] C.V. Jakowatz Jr., D.E. Wahl, P.H. Eichel, D.C. Ghiglia, P.A. Thompson, SpotlightMode Synthetic Aperture Radar: A Signal Processing Approach, Springer, 1996.

[40] D.C. Munson Jr., J.C. O'Brien, W.K. Jenkins, A tomographic formulation of spotlight-mode synthetic aperture radar, Proc. IEEE 71 (8) (1983) 917-925.

[41] M. Cetin, W.C. Karl, Feature-enhanced synthetic aperture radar image formation based on nonquadratic regularization, IEEE Trans. Image Process. 10 (4) (2001) 623-631.

[42] R. Baraniuk, Compressive sensing, IEEE Signal Process. Mag. 24 (4) (2007) 118121.

[43] G. Hennenfent, F.J. Herrmann, Simply denoise: wavefield reconstruction via jittered undersampling, Geophysics 73 (3) (2008) v19-v28.

[44] R. Tibshirani, Regression shrinkage and selection via the lasso, J. Roy. Statist. Soc. Ser. B 58 (1996) 267-288.

[45] W. Boyd, L. Vandenberghe, Convex Optimization, Cambridge University Press, 2004

[46] M. Lustig, D. Donoho, D.M. Pauly, Sparse MRI: the application of compressed sensing for rapid MR imaging, Magn. Reson. Med. 58 (6) (2007) 1182-1195.

[47] E. Candès, J. Romberg, Practical signal recovery from random projections, in: Computational Imaging III: Proc. SPIE International Symposium on Electronic Imaging, San Jose, 2005, pp. 76-86.

[48] L.I. Rudin, S. Osher, E. Fatemi, Nonlinear total variation based noise removal algorithms, Physica D 60 (1992) 259-268. 
[49] M.M. Bronstein, A.M. Bronstein, M. Zibulevsky, H. Azhari, Reconstruction in diffraction ultrasound tomography using nonuniform FFT, IEEE Trans. Med. Imaging 21 (11) (2002) 1395-1401.

[50] K.T. Block, M. Uecker, J. Frahm, Undersampled radial MRI with multiple coils. Iterative image reconstruction using a total variation constraint, Magn. Reson. Med. 57 (2007) 1086-1098.

[51] D.P. Bertsekas, Nonlinear Programming, Athena Scientific, 1995.

[52] J.F. Cai, S. Osher, Z. Shen, Split Bregman methods and frame based image restoration, Multiscale Model. Simul. 8 (2) (2009) 337-369.

[53] J.F. Cai, S. Osher, Z. Shen, Linearized Bregman iterations for compressed sensing, Math. Comp. 78 (267) (2009) 1515-1536.

[54] J.F. Cai, S. Osher, Z. Shen, Linearized Bregman iterations for frame-based image deblurring, SIAM J. Imaging Sci. 2 (1) (2009) 226-252.

[55] The MathWorks, Inc., Natick, MA, USA.

[56] K. Goto, R.A. Van de Geijn, Anatomy of high-performance matrix multiplication, ACM Trans. Math. Software 34 (3) (2008), Article 12.

[57] J.A. Gunnels, G.M. Henry, R.A. Van de Geijn, A family of high-performance matrix multiplication algorithms, in: ICCS 2001, in: Lecture Notes in Computer Science, vol. 2073, 2001, pp. 51-60.

[58] The Sensor Data Management System MSTAR public data, http://www.sdms. afrl.af.mil.

[59] S. Samadi, M. Cetin, M.A. Masnadi-Shirazi, Sparse representation based synthetic aperture radar imaging, Radar Sonar Navig. 5 (2) (2011) 182-193.

[60] M. C̣etin, W.C. Karl, D.A. Castañon, Feature enhancement and ATR performance using nonquadratic optimization-based SAR imaging, IEEE Trans. Aerospace Electron. Syst. 39 (4) (2003) 1375-1395.
Salih Uḡur was born in 1971 in Ankara, Turkey. He received the B.Sc. degree in Electrical and Electronics Engineering from the Middle East Technical University, Ankara, Turkey, in 1993 and the M.S. degree in Electrical and Electronics Engineering from Bilkent University, Ankara, Turkey, in 1996. He currently works for Meteksan Savunma and is a Ph.D. candidate in the Electrical and Electronics Engineering Department of Bilkent University, Ankara, Turkey. His research interests include radar signal processing, remote sensing and compressed sensing.

Orhan Arıkan was born in 1964 in Manisa, Turkey. In 1986, he received his B.Sc. degree in Electrical and Electronics Engineering from the Middle East Technical University, Ankara, Turkey. He received both his M.S. and Ph.D. degrees in Electrical and Computer Engineering from the University of Illinois, Urbana-Champaign, in 1988 and 1990, respectively Following his graduate studies, he was employed as a Research Scientist at Schlumberger-Doll Research Center, Ridgefield, CT. In 1993 he joined the Electrical and Electronics Engineering Department of Bilkent University, Ankara, Turkey. Since 2006, he is a full professor at Bilkent University His current research interests include statistical signal processing, timefrequency analysis and remote sensing. Dr. Arlkan has served as the chairman of Turkey chapter of IEEE Signal Processing Society and president of IEEE Turkey Section. Since 2011, he is serving as the chairman of the Department of Electrical and Electronics Engineering. 